\title{
Effect of Resonant Magnetic Perturbation Field on Energetic Ion Behavior in the Large Helical Device
}

\author{
Kunihiro OGAWA ${ }^{1,2)}$, Sergey A. BOZHENKOV ${ }^{3)}$, Simppa ÄKÄSLOMPOLO ${ }^{3)}$, \\ Mitsutaka ISOBE ${ }^{1,2)}$, Hideo NUGA ${ }^{1)}$, Ryosuke SEKI ${ }^{1,2)}$, Shuji KAMIO ${ }^{1)}$, Yutaka FUJIWARA ${ }^{1)}$, \\ Takeo NISHITANI ${ }^{1)}$, Masaki OSAKABE ${ }^{1,2)}$ and the LHD Experiment Group ${ }^{1)}$ \\ ${ }^{1)}$ National Institute for Fusion Science, National Institutes of Natural Sciences, Toki 509-5292, Japan \\ ${ }^{2}$ SOKENDAI (The Graduate University for Advanced Studies), Toki 509-5292, Japan \\ ${ }^{3)}$ Max-Planck-Institute for Plasma Physics, Greifswald, Germany
}

(Received 24 June 2019 / Accepted 2 September 2019)

\begin{abstract}
A study of the resonant magnetic perturbation (RMP) effect on transit beam ion behavior is performed using the total neutron emission rate $\left(S_{\mathrm{n}}\right)$ measurement of the deuterium plasma in the Large Helical Device. We conducted no RMP field, one-half RMP field, and full RMP field discharges and compared $S_{\mathrm{n}}$ that reflects the global beam ion confinement information. It is determined that owing to the RMP field, $S_{\mathrm{n}}$ decreased by approximately 15 - 30\%. Numerical calculations based on the classical confinement of beam ions were performed to investigate the bulk plasma parameter effect on $S_{\mathrm{n}}$. The calculated $S_{\mathrm{n}}$ shows that the degradation of $S_{\mathrm{n}}$ by RMP is mainly caused by the degradation of the electron temperature owing to island formation which results in a shorter slowing down time of beam ions.
\end{abstract}

(C) 2019 The Japan Society of Plasma Science and Nuclear Fusion Research

Keywords: energetic ion confinement, resonant magnetic perturbation field, Large Helical Device, neutron diagnostics

DOI: $10.1585 /$ pfr.14.1202159

To realize a nuclear fusion reactor, it is essential to produce high-performance plasma. However, the performance is limited by magnetohydrodynamic modes. To avoid these modes, the application of three-dimensional resonant magnetic perturbation (RMP) has been widely used [1]. However, RMP may induce the degradation of fast ion confinement by breaking the symmetry of the magnetic field. In the Large Helical Device (LHD), the effect of the RMP field on the beam ion losses has been studied in hydrogen plasmas with a fast ion loss detector (FILD) [2]. It has been reported that the beam ion-loss rate to the FILD increased in wide energy and wide pitch angle ranges. Although FILD measurements shows the effect of RMP on the beam ion loss, the RMP effect on confined beam ion remains unclear because the FILD can only measure the local beam ion loss. In the deuterium operation of LHD, owing to the intensive neutral beam (NB) injection, neutrons from the NB-heated plasma primarily originate from the fusion reaction between the bulk plasma and beam ions [3]. Therefore, we can obtain information on the global beam ion behavior by the total neutron emission rate $\left(S_{\mathrm{n}}\right)$ measurement. This paper reports the RMP effect on the global beam ion behavior by neutron diagnostics.

LHD is equipped with three negative-source-based tangential NB injectors (i.e., NB1, NB2, and NB3) and positive-source-based perpendicular neutral beam injections (i.e., NB4 and NB5). NB1, NB2, and NB3 primar-

author'se-mail: kogawa@nifs.ac.jp ily create passing transit beam ions with energies of up to $180 \mathrm{keV}$, whereas helically-trapped beam ions with energies of $60-80 \mathrm{keV}$ are mainly created by NB4 and NB5. To measure $S_{\mathrm{n}}$, an absolutely-calibrated neutron flux monitor installed on the top of the LHD is used [4]. Here the typical error bar originating from the absolute calibration and pulse statistics in $S_{\mathrm{n}}$ of $\sim 10^{14} \mathrm{n} / \mathrm{s}$ is approximately $10 \%$. The RMP field is externally applied by a local island divertor coil consisting of 10 pairs of copper coils installed outside the vacuum vessel of the LHD [5].

The beam ion confinement study was performed with a toroidal magnetic field strength $B_{\mathrm{t}}$ of $2.75 \mathrm{~T}$ (the direction of the toroidal field is counterclockwise from the top) and a preset magnetic axis position $R_{\mathrm{ax}}$ of $3.6 \mathrm{~m}$. Here, co-going transit beam ions are injected by NB1 and NB3, whereas counter-going transit beam ions are injected by NB2. We conducted plasma discharges with no RMP field, one-half RMP field strength, and full RMP field strength. Figure 1 shows the typical time evolution of the injection power $\left(P_{\text {inj }}\right)$ of ECRH and NBs, together with the time evolution of the electron temperature at the plasma center $\left(T_{\mathrm{e} 0}\right)$ and the line-averaged electron density $\left(n_{\mathrm{e} \_a v g}\right)$ in each shot. In this discharge, ECRH and NBs are sequentially injected into the plasma. Here, NB4 is used as a diagnostic beam for the ion temperature measurement. The acceleration voltages of NB1, NB2, and NB3 are $180 \mathrm{keV}, 150 \mathrm{keV}$, and $170 \mathrm{keV}$, respectively. Here, in shot number 146408 and shot number 146409, RMP with a constant amplitude 


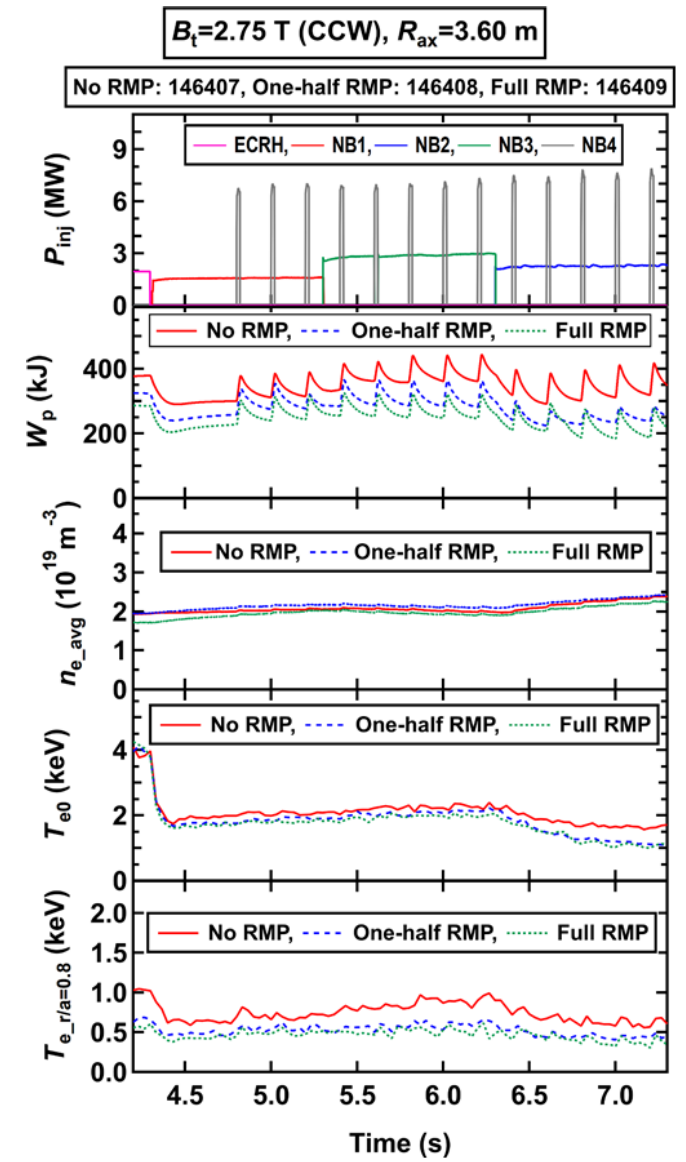

Fig. 1 Time trace of discharges with and without RMP. Each NB is sequentially injected into the plasma with/without the RMP field. $W_{\mathrm{p}}$ degrades by $20-30 \%$ owing to RMP because of the magnetic island formation.

is applied throughout the discharge. Note that the amplitudes of magnetic field perturbation in one-half RMP and full RMP cases at the magnetic axis position are $1.2 \mathrm{mT}$ and $2.3 \mathrm{mT}$, respectively. In addition, there was almost no difference in $T_{\mathrm{e} 0}$ and $n_{\mathrm{e} \_ \text {avg. }}$. However, diamagnetic energy $\left(W_{\mathrm{p}}\right)$ decreased owing to RMP. This is attributed to the formation of a magnetic island with a poloidal mode number of 1 and a toroidal mode number of 1 at $r / a \sim 0.8$ with an island width of $r / a \sim 0.1$. For example, $T_{\mathrm{e}}$ at $r / a=0.8$ $\left(T_{\mathrm{e}_{-} \mathrm{r} / \mathrm{a}=0.8}\right)$ is lower in RMP cases compared with the no RMP case. Therefore, plasmas have a relatively narrow $T_{\mathrm{e}}$ profile in the $r / a<0.8$ region with RMP.

The time evolution of $S_{\mathrm{n}}$ measured by NFM is shown in Fig. 2. The absolute value of $S_{\mathrm{n}}$ is different in each NB because of the difference in the acceleration voltage and injection power. This experiment yields the degradation of $S_{\mathrm{n}}$ owing to RMP field application. In the one-half RMP case, $S_{\mathrm{n}}$ decreases by $\sim 15 \%$ for NB1, $30 \%$ for NB2, and $\sim 20 \%$ for NB3 compared with that of the no RMP case. The absolute value of $S_{\mathrm{n}}$ is comparable with $S_{\mathrm{n}}$ for the cases of one-half RMP and full RMP. The calculation of $S_{\mathrm{n}}$ by the FBURN code based on the classical confine-
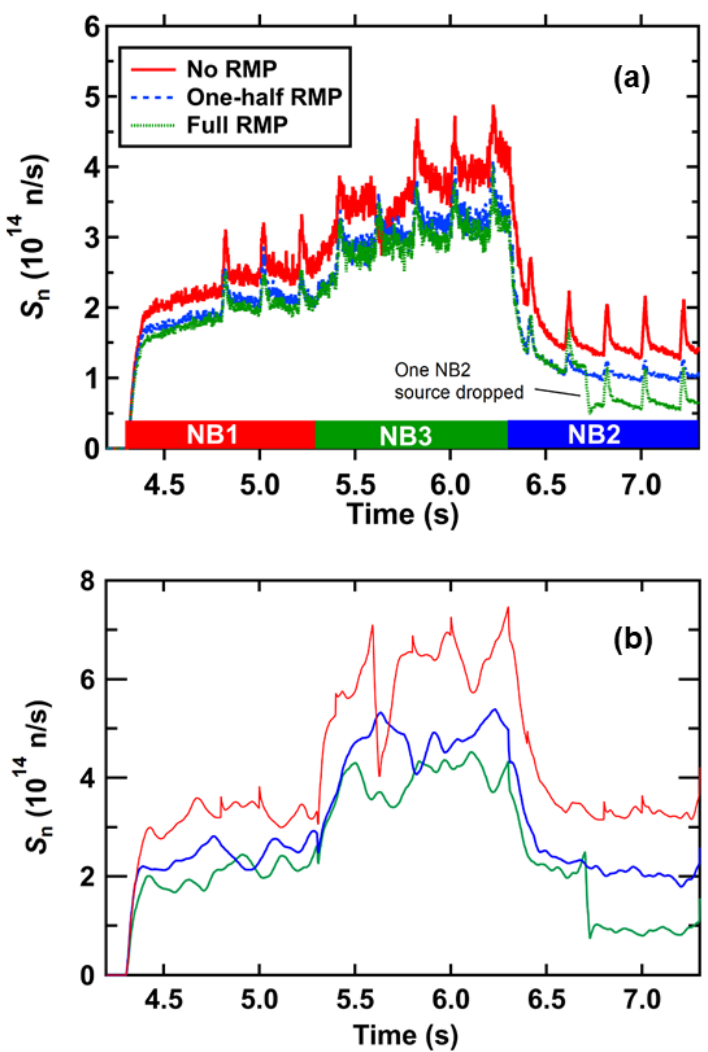

Fig. 2 (a) Time evolution of $S_{\mathrm{n}}$ measured in the experiments. The pulsed increase in $S_{\mathrm{n}}$ is due to the diagnostic beam (NB4) injection. (b) Time evolution of $S_{\mathrm{n}}$ calculated by the FBURN code.

ment of the beam ion [6] using the experimental data is performed to investigate the effect of the bulk plasma parameter change owing to RMP on $S_{\mathrm{n}}$. In the calculation, beam ions are assumed to be slowed down at the deposited position; effective charge of 2 is assumed, and no radial diffusion is included. The absolute value of $S_{\mathrm{n}}$ obtained in the calculation is approximately 1.5 times higher than that of the calculated $S_{\mathrm{n}}$, as reported in Ref. [6]. The calculation results show that $S_{\mathrm{n}}$ decreases by approximately $15 \%$ for NB1, 30\% for NB2, and $20 \%$ for NB3 owing to RMP. It should be pointed out that $S_{\mathrm{n}}$ in the one-half RMP amplitude is almost the same as $S_{\mathrm{n}}$ in the full RMP amplitude, as measured in the experiments. The result suggests that the degradation of $S_{\mathrm{n}}$ is mainly due to the degradation of the electron temperature owing to the island formation because lower electron temperature induces shorter slowing down time of beam ions. The detailed analysis with a focus on the effect of RMP on the beam ion orbit will be performed.

This work is supported by the NINS program of Promoting Research by Networking among Institutions (Grant Number 01411702).

[1] T. Hender et al., Nucl. Fusion 32, 2091 (1992).

[2] K. Ogawa et al., Plasma Fusion Res. 9, 03402097 (2014). 
[3] M. Osakabe et al., Fusion Sci. Technol. 72, 199 (2017).

[4] M. Isobe et al., Rev. Sci. Instrum. 85, 11E114 (2014).

[5] T. Morisaki et al., Fusion Sci. Technol. 58, 232 (2010).
[6] K. Ogawa et al., Plasma Phys. Control. Fusion 60, 095010 (2018). 